\title{
Magnetic core shell nanoparticles trapping in a microdevice generating high magnetic gradient $\dagger+$
}

\author{
Bruno Teste, ${ }^{a}$ Florent Malloggi, ${ }^{b}$ Anne-Laure Gassner, ${ }^{c}$ Thomas Georgelin, ${ }^{d}$ Jean-Michel Siaugue, ${ }^{d}$ \\ Anne Varenne, ${ }^{a}$ Hubert Girault ${ }^{c}$ and Stéphanie Descroix ${ }^{* a}$
}

\author{
Received 15th October 2010, Accepted 15th December 2010 \\ DOI: $10.1039 / \mathbf{c 0 l c 0 0 5 1 0 j}$
}

\begin{abstract}
Magnetic core shell nanoparticles (MCSNPs) $30 \mathrm{~nm}$ diameter with a magnetic weight of 10\% are usually much too small to be trapped in microfluidic systems using classical external magnets. Here, a simple microchip for efficient MCSNPs trapping and release is presented. It comprises a bed of micrometric iron beads ( $6-8 \mu \mathrm{m}$ diameter) packed in a microchannel against a physical restriction and presenting a low dead volume of $0.8 \mathrm{~nL}$. These beads of high magnetic permeability are used to focus magnetic field lines from an external permanent magnet and generate local high magnetic gradients. The nanoparticles magnetic trap has been characterised both by numerical simulations and fluorescent MCSNPs imaging.

Numerical simulations have been performed to map both the magnetic flux density and the magnetic force, and showed that MCSNPs are preferentially trapped at the iron bead magnetic poles where the magnetic force is increased by 3 orders of magnitude. The trapping efficiency was experimentally determined using fluorescent MCSNPs for different flow rates, different iron beads and permanent magnet positions. At a flow rate of $100 \mu \mathrm{L} \mathrm{h}^{-1}$, the nanoparticles trapping/release can be achieved within $20 \mathrm{~s}$ with a preconcentration factor of 4000 .
\end{abstract}

\section{Introduction}

Microfluidics is a field that has received growing interest during the last years. Original microdevices have been developed for a wide range of applications such as diagnosis, cell and tissue investigation. Microfluidics combines the advantages of low sample and reagent consumption, high throughput analysis and integration capability. In parallel, the potential of magnetic particles has been intensively demonstrated during the last decade. Magnetic particles are considered as versatile and powerful tools at the macro and micro-scale. They have been widely used for chemical, biological and biochemical applications. ${ }^{1-4}$ Magnetic particles are commercially available and their surface chemistry can be tuned to achieve high specific binding capacity while avoiding non-specific adsorption. As recently

${ }^{a}$ Physicochimie des Electrolytes, Collö̈des et Sciences Analytiques (PECSA), UMR 7195 CNRS-ESPCI-ENSCP, France. E-mail: stephanie.descroix@espci.fr

${ }^{b} M M N$ - UMR 7083 - Gulliver CNRS-ESPCI, Paris, France

'Laboratoire d'Electrochimie Physique et Analytique, Ecole Polytechnique Fédérale de Lausanne, Station 6, CH-1015 Lausanne, Switzerland

${ }^{\text {} P h y s i c o c h i m i e}$ des Electrolytes, Collö̈des et Sciences Analytiques (PECSA), UMR 7195 CNRS-Paris6-ESPCI, Paris, France

$\dagger$ Electronic supplementary information (ESI) available: Electronic supplementary information. See DOI: $10.1039 / \mathrm{c} 0 \mathrm{lc} 00510 \mathrm{j}$

* Published as part of a LOC themed issue dedicated to French Research: Guest Editor Professor Jean-Louis Viovy. highlighted by Gijs et al., ${ }^{5}$ the combination of microfluidics and magnetic particles has led to highly multidisciplinary research with a wide range of applications such as cell handling and separation, nucleic acid processing, bioassays and catalysis. Promising developments based on the integration of magnetic particles in microdevices have already been described and further are expected. Pamme has also reported that different operation units can be achieved by magnetic forces, ${ }^{6}$ like mixing and pumping, incorporation of valves, trapping and transporting, separation and sorting, magnetic plug formation as solid support for bioassay. ${ }^{7,8}$ Indeed, magnetic particles provide a large surface to volume ratio while avoiding the complex development of monolith as stationary phase or the integration of physical restriction to pack beads. Moreover, as previously mentioned, their surface chemistry can be adapted to the analytical requirements. In this context, particle trapping is considered as a crucial issue. Different strategies have been developed to capture magnetic beads. The most widely used method consists in placing a permanent magnet, ${ }^{9}$ or an electromagnet, ${ }^{10}$ outside the microchannel. The need for high magnetic flux density $(\boldsymbol{B})$ until 1 Tesla $(\mathrm{T})$ is provided by permanent magnets like neodymium iron boron magnets $(\mathrm{NdFeB})$ whereas electromagnets generate lower $\boldsymbol{B}$. The latter are preferred if $\boldsymbol{B}$ has to be tuned or switched on and off rapidly. For both types of magnets, $\boldsymbol{B}$ decreases as the distance from the magnet increases. This simple approach led to many assay applications ${ }^{11-13}$ A second 
approach consists of integrating soft magnetic structures into microdevices to locally increase the magnetic field. The magnetic structures are usually made of ferromagnetic material like iron, ${ }^{14}$ nickel, ${ }^{15}$ or permalloy. ${ }^{16}$ These materials generate a high magnetic field when they are submitted to an external magnetic excitation and a low remanent field can remain when the external permanent magnet is removed. This microfabrication approach requires several complex fabrication steps using different techniques like polymer photo-ablation, ${ }^{14}$ or a combination of lithography and etching. ${ }^{17}$

Finally, different studies have shown that it is quite easy to capture efficiently magnetic particles in microdevices of various geometries. ${ }^{18}$ Nevertheless, in the literature, most of the particles magnetically trapped were micrometric since the magnetic force on a particle is proportional to its volume and relative magnetic weight. Lately, nanoparticles became an interesting tool for various biological technologies. ${ }^{1920}$ They present a higher surface to volume ratio and they have been already used for various applications from single molecule study, ${ }^{21}$ to cell manipulation. ${ }^{22}$ At the macroscale, special attention has been paid to separate ferromagnetic and paramagnetic nanomaterials from various matrices by high-gradient magnetic separation (HGMS). ${ }^{23}$ For that purpose, high magnetic field gradients are achieved by using small magnetic elements such as stainless-steel wool or wire. Based on this technique, the magnetic cell sorting technology (MACS) is currently widely used to retain cells bound to superparamagnetic nanoparticles prior to flow cytometry. The main limitation of the MACS system is related to high volume consumption due to the macrosize of the system. Very few papers report on the development of sub-100 nm nanoparticles trapping at the microscale. Only sophisticated microdevices have been developed to trap such nanoparticles. Recently, Donolato et al. ${ }^{24}$ reported on a device with a corner-shaped permalloy nanostructure terminated by two circular disks to trap and detect a small number of nanoparticles.
In this paper, we present a simple and efficient microdevice based on HGMS principle that avoids complicated and time consuming processes for the trapping of colloidal $30 \mathrm{~nm}$ magnetic core shell nanoparticles (MCSNPs). Micrometric iron beads $(6-8 \mu \mathrm{m})$ packed by a physical restriction within a microchannel were used to develop a miniaturized magnetic chamber, which offers a very small dead volume and generate a very high $\boldsymbol{B}$ gradient. An external permanent magnet was used to magnetize and therefore address the magnetic flux density through the ferromagnetic iron beads (Fig. $1 \mathrm{a}, \mathrm{b}$ ). Due to the non-uniform magnetic field achieved, the nanoparticles migrate by magnetophoresis and are finally trapped within the magnetic chamber. ${ }^{25}$ The magnetic chamber has a volume $10^{7}$ times smaller than those used in conventional MACS cell sorting. It has been investigated both by finite element numerical simulations and fluorescent MCSNPs imaging. Numerical simulations were used to calculate the $\boldsymbol{B}$ value as well as its spatial distribution. The same study was performed to map the magnetic force $\boldsymbol{F}_{\boldsymbol{m a g}}$ in order to evaluate the zone where the MCSNPs should be preferentially trapped. To optimize the microdevice development, the influence of magnetic chamber elements like beads size, nature and bead plug length on $\boldsymbol{F}_{\boldsymbol{m a g}}$ was also evaluated. The feasibility of developing such a magnetic chamber was finally experimentally validated using fluorescent MCSNPs. Different parameters such as bead nature constituting the magnetic chamber, magnet position and flow rate were investigated.

\section{Materials and methods}

\section{Microsystem fabrication and set up}

The microdevice was fabricated in two main steps: master fabrication and replica moulding. Master fabrication was performed using the standard multilayer soft lithography method. ${ }^{26,27}$ The microstructures (channel and restriction) were designed in a computer aided design (CAD) program.

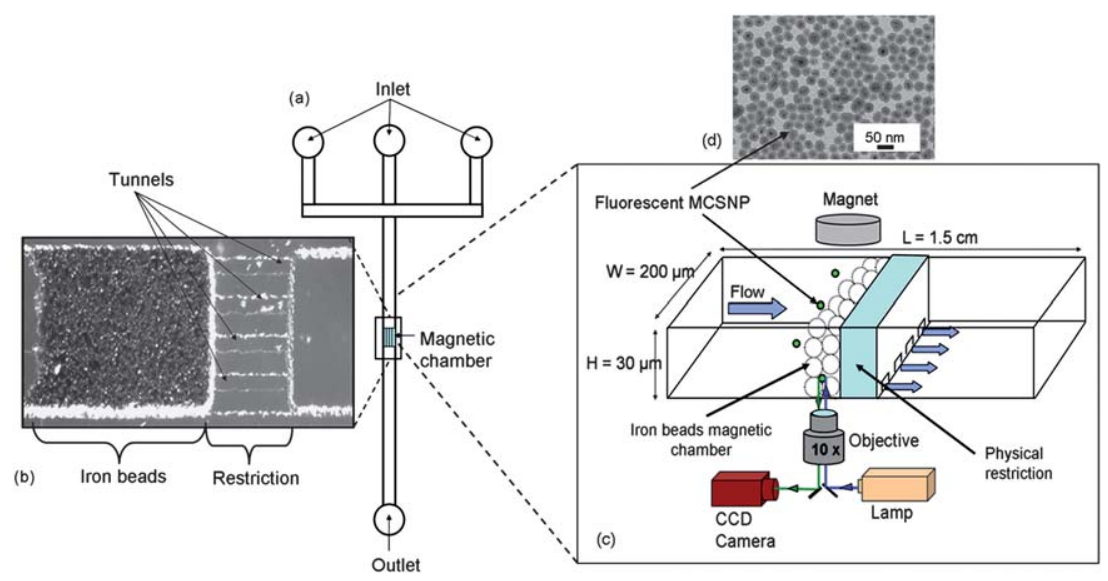

Fig. 1 Experimental microdevice and set up dedicated to MCSNPs trapping and release. (a) Scheme of the device. It is composed of an inlet, outlet and a physical restriction located in the middle of the microchannel. (b) Picture of magnetic chamber (top view). 6-8 $\mu \mathrm{m}$ iron beads were packed against physical restriction; magnetic plug length is about $300 \mu \mathrm{m}$. (c) Scheme of the magnetic chamber and set up used for visualization of MCSNPs trapping and release. Permanent magnet was placed on the top of the microchip to magnetize iron beads magnetic chamber. Excitement light from mercury lamp was focused into the magnetic plug area using microscope objective (x 10) and fluorescent light emitted from MCSNPs was collected using CDD camera. The fluids pass through 4 tunnels of $3 \mu \mathrm{m}$ in height and $25 \mu \mathrm{m}$ in width. (d) Transmission electron microscopy image of MCSNPs made of maghemite nanoparticles (dark) encapsulated in silica shell (dark grey). 
Transparencies of the CAD-generated patterns were then created using commercial services. The first layer was obtained by spin coating the negative SU-8 2002 photoresist (Microchem, viscosity $7.5 \mathrm{cSt}$ ) at $2000 \mathrm{rpm}$. The manufacturer predicts a layer height of $2.4 \mu \mathrm{m}$. The restriction design was insulated $(435 \mathrm{~nm}$, $7 \mathrm{~s})$ onto the wafer and then developed. We measured the height of the restriction with a stylus profiler Dektak4M (Veeco). The measured restriction height $(3 \mu \mathrm{m})$ was in good agreement with the photoresist manufacturer predictions. On the same wafer we span coat the second layer. We used the negative SU-8 2015 photoresist (Microchem, viscosity 1250cSt) at $1500 \mathrm{rpm}$. The channel design is then aligned with a mask aligner MJB4 (SUSS MicroTec) onto the restrictions and then insulated (435 nm, 10 $\mathrm{s})$. The wafer is developed and the height measured. The second layer height is approximately $30 \mu \mathrm{m}$. The polydimethylsiloxane Sylgard 184 elastomer (PDMS, Dow Corning) was poured and further cured on a SU-8 mold (Microchem). Holes were punched for the inlets and the PDMS replicates were sealed to glass slides following oxygen plasma treatment at 200 mTorr for $30 \mathrm{~s}$ using plasma cleaner (Harrick scientific).

Spherical iron beads ( $7 \pm 0.6 \mu \mathrm{m}$, Good Fellow) offering a saturation magnetization of about $1.1 \times 10^{6} \mathrm{~A} \mathrm{~m}^{-1}$ were used for the magnetic chamber development. Four different permanent magnets (Chen Yang) were used: 3 cylindrical $\mathrm{NdFeB}$ magnets (magnet 1: $2 \mathrm{~cm}$ diameter and $1 \mathrm{~cm}$ high; magnet $3: 0.5 \mathrm{~cm}$ diameter and $1.2 \mathrm{~cm}$ high; magnet $4: 0.4 \mathrm{~cm}$ diameter and $0.3 \mathrm{~cm}$ high) and one $\mathrm{NdFeB}$ square magnet (magnet 2: $2 \mathrm{~cm}$ long and 0.4 $\mathrm{cm}$ thick). All the magnets present a magnetic remanence of $1.3 \mathrm{~T}$. The formation of the magnetic bead plug is performed under a sufficient flow rate in order to avoid the iron bead sedimentation. The samples were injected using syringes of different volumes. The latter were connected to the microsystem inlet by a capillary tube. The flow rate was controlled by a syringe pump (Kd scientific).

Microchip analyses were monitored by an IX-71 inverted fluorescence microscopic system (Olympus) equipped with spectral filters, 460-490 $\mathrm{nm}$ and a $100 \mathrm{~W}$ mercury lamp (Olympus). A CCD camera $1388 \times 1038$ pixels Pike (RD Vision) was mounted on the microscope and Hiris software (RD Vision) was used for camera control and image processing.

\section{Fluorescent magnetic core shell nanoparticle synthesis}

Briefly, maghemite nanoparticles (mean physical diameter $7 \mathrm{~nm}$ ) were prepared by co-precipitation of $\mathrm{Fe}^{2+}$ and $\mathrm{Fe}^{3+}$ ions under alkaline conditions as described by Massart. ${ }^{28}$ Nanoparticles were coated by citrate anions and dispersed in water. ${ }^{29}$ These maghemite nanoparticles were encapsulated in silica shells. A first silica shell was prepared by condensation of tetraethoxyorthosilicate (TEOS, Sigma), ${ }^{30}$ in ethanolic medium in the presence of ammonia as catalyst. The fluorescein dye bearing an isothiocyanate functional group (FITC, Sigma) was previously coupled to the amino group of 3-aminopropyl-triethoxysilane (APTS, Sigma) through an addition reaction. Stable fluorescein dye was then incorporated within the first silica shell by co-hydrolyzing with TEOS. The latter was then functionalized by simultaneous condensation of APTS and a silica polyethylene glycol-derived compound, 2-[methoxy(polyethyleneoxy)propyl]trimethoxysilane (PEOS, ABCR). The concurrent addition of a small amount of TEOS resulted in the formation of a crosslinked silica shell. ${ }^{31,32}$ The PEOS/APTS molar ratio used was $3: 1$. The reaction of silica condensation was carried out overnight and the particle suspension was destabilized by diethyl ether. A red pellet was formed and separated by magnetic settling. The pellet was washed twice with a mixture of diethyl ether and ethanol $(15: 1)$ and redispersed in $10 \mathrm{mM}$ MOPS buffer ( $\mathrm{pH} 7.5$ ). This colloidal solution of amino-PEOS functionalized MCSNP (approximately $3.3 \times 10^{14} \mathrm{MCSNP} / \mathrm{mL}$ ) was stable for months. The value of saturation magnetization obtained for core-shell nanoparticles was similar to maghemite nanoparticles, approximately equal to 3 $\times 10^{5} \mathrm{~A} \mathrm{~m}^{-1}$.

\section{Magnetic core shell nanoparticle trapping and release}

The magnetic chamber was built by injecting iron beads $(5 \mathrm{mg}$ $\mathrm{mL}^{-1}$ ) solubilized in PBS-Tween $0.1 \%(\mathrm{v} / \mathrm{v})$ using a $100 \mu \mathrm{L}$ syringe until the desired plug length was obtained. Then fluorescent MCSNP $\left(6.2 \times 10^{13} \mathrm{MCSNPs} / \mathrm{mL}\right)$ were injected at various flow rates (from 10 to $100 \mu \mathrm{L} \mathrm{h}^{-1}$ ) using a $1 \mathrm{~mL}$ syringe. External magnets are placed from 0.5 to $2 \mathrm{~cm}$ from the top of the channel. Finally the MCSNPs were released by manually removing the external magnet.

The capture capacity was evaluated as follows. A solution of fluorescent nanoparticles at fixed concentration was flowed through the channel at a given flow rate. The fluorescent signal was measured in a zone located after the physical restriction. During the nanoparticles trapping the fluorescence remains null. When the magnetic chamber saturation is reached, the fluorescence increases. Knowing the time required to reach the saturation, the device dead volume as well as the nanoparticles concentration, the amount of trapped nanoparticles can be estimated.

Concerning the elution efficiency, fluorescent signal was measured in the plug before the trapping and $15 \mathrm{~s}$ after the MCSNPs release. Repeatability of MCSNPs trapping was evaluated by measuring the peak area following MCSNPs elution from the magnetic chamber.

\section{Results and discussion}

\section{Microdevice and magnetic chamber design}

Due to their low volume and relative magnetic weight, magnetic nanoparticles cannot be trapped by the usual strategies applied to micrometric beads that consist in placing a magnet near the microchannel. High magnetic gradients are required to perform an efficient trapping of magnetic nanoparticles. Based on the principle of high magnetic gradient separator (HGMS), a microdevice (Fig. 1a, c) dedicated to colloidal magnetic nanoparticles trapping and release was developed. For that purpose, a monochannel $1.5 \mathrm{~cm}$ long, $200 \mu \mathrm{m}$ wide and $30 \mu \mathrm{m}$ high was microfabricated. In this study, the HGMS was composed of an external permanent magnet coupled to an internal magnetic chamber filled with micrometric magnetic iron beads (Fig. 1b). Indeed, for a material located in a magnetic field $\boldsymbol{H}$ generated by a permanent magnet, the magnetic flux density $\boldsymbol{B}$, will be defined $\mathrm{as}^{33}$ :

$$
\boldsymbol{B}=\mu_{0}(\boldsymbol{H}+\boldsymbol{M})
$$

Where $\mu_{0}$ is the permeability of free space and $\boldsymbol{M}$ the magnetization of the material induced by $\boldsymbol{H}$. 
A superparamagnetic particle is submitted to a magnetic force $\boldsymbol{F}_{\boldsymbol{m a g}},{ }^{34}$ described as follows:

$$
\boldsymbol{F}_{\text {mag }}=\frac{V \Delta \chi}{\mu_{0}}(\boldsymbol{B} \cdot \nabla) \boldsymbol{B}
$$

$\boldsymbol{F}_{\boldsymbol{m a g}}$ depends on the volume of the particle $(V)$, the difference in magnetic susceptibility $(\Delta \chi)$ between the particle and the surrounding medium as well as the magnitude and gradient of $\boldsymbol{B}$. Homogeneous $\boldsymbol{B}$ will align the particles in the direction of the magnetic field, but a $\boldsymbol{B}$ gradient is required to exert a translational force on a magnetic particle.

In a microfluidic system, a particle is also submitted to the gravitational force $\boldsymbol{F}_{\boldsymbol{g}}$ and to the hydrodynamic drag force $\boldsymbol{F}_{\boldsymbol{d r a g}}$ defined in eqn (3). According to the volume and colloidal nature of the MCSNPs (Fig. 1d), $\boldsymbol{F}_{\boldsymbol{g}}$ was neglected compared to $\boldsymbol{F}_{\boldsymbol{m a g}}$ and $\boldsymbol{F}_{\text {drag }}$,

$$
\boldsymbol{F}_{\text {drag }}=6 \pi \eta R \Delta v
$$

with $\eta$ the fluid viscosity, $R$ the radius of the particle and $\Delta v$ the linear velocity of the particle compared to the surrounding fluid velocity.

As previously mentioned, a high $\boldsymbol{B}$ magnitude and gradient are required to trap magnetic nanoparticles. However, the $\boldsymbol{B}$ value decreases with the square of the distance from the magnetic source or magnetized material. ${ }^{33}$ To limit this effect, ferromagnetic iron beads have been integrated directly in the microchannel in order to increase the local value of $\boldsymbol{B}$. Iron beads were packed in the microchannel against a physical restriction made of tunnels $3 \mu \mathrm{m}$ high and $25 \mu \mathrm{m}$ wide allowing fluids to pass. This approach avoids complicated and time consuming microfabrication processes.

A high $\boldsymbol{B}$ magnitude and gradient are generated by exposing the ferromagnetic iron beads to a magnetic field produced by an external $\mathrm{NdFeB}$ permanent magnet. The magnetic field lines are expected to be concentrated through the iron beads due to their high magnetic permeability and thus to locally increase $\boldsymbol{B}$ and consequently $\boldsymbol{F}_{\boldsymbol{m a g}}$. Different permanent magnets have been used, all of them presenting a width much larger than the length of the iron beads plug. In this case, the iron beads plug was located in the middle of the magnet, where the magnetic field lines are parallel near the surface and $\boldsymbol{B}$ is homogeneous.

Spheric and small sized beads presenting low polydispersity (6-8 $\mu \mathrm{m})$ were preferred for several reasons. A low polydispersity should favour iron beads plug formation with a good reproducibility. Small diameter beads allow the formation of a magnetic chamber with a low dead volume. Considering a plug length of $300 \mu \mathrm{m}$ and a filling factor approximated to 0.45 by fluorescent liquid filling measurements, the dead volume of the magnetic plug was roughly $0.8 \mathrm{~nL}$. Moreover, high polydispersity beads increase the nanoparticle band broadening, as Eddy diffusion contributes to paths of different lengths through the magnetic beads plug.

Magnetic beads arrangement in microdevices has already been studied. ${ }^{35,36}$ In homogeneous magnetic fields, magnetic beads are aligned in columns following the field lines direction. In the present microdevice, despite the presence of homogeneous $\boldsymbol{B}$ in the centre of the magnet pole, no significant effect of the permanent magnet was observed on the beads arrangement since the latter are packed against a physical restriction and the magnetic field was only applied after beads packing.

\section{Magnetic flux density and magnetic force mapping}

Finite elements numerical simulations were performed in 2D geometries, as shown in Fig. 2, to characterize $\boldsymbol{B}$ and $\boldsymbol{F}_{\text {mag }}$. Using a centimetre-sized permanent magnet, experimental conditions cannot be directly transposed to simulation due to software limitations. At the microchannel scale, the magnetic field lines can be considered as parallel on the $y$-axis using a cylindrical magnet $2 \mathrm{~cm}$ in diameter and $1 \mathrm{~cm}$ high (magnet 1). In order to reflect the present experimental conditions, simulations were thus performed with two magnets in attraction since the magnetic field lines between the two magnets are parallel, numerical model and parameters are described in supplementary information (SI 1, 2, 3) $\dagger$. Finally, even if results from the simulation cannot be quantitatively compared with experimental results, simulations are used to validate the principle of this microdevice as well as to describe the $\boldsymbol{F}_{\boldsymbol{m a g}}$ gain, to depict $\boldsymbol{B}$ and $\boldsymbol{F}_{\boldsymbol{m a g}}$ spatial distribution and to optimize the magnetic chamber development.

The first aim of these simulations was to evaluate the effect of an iron bead column on $\boldsymbol{B}$ within the microchannel. For that purpose, two configurations have been compared: in the presence and absence of iron beads within the microchannel respectively. Considering a 2D system, $\boldsymbol{B}$ vectors have two components ( $x$ and $y$ ) and are tangential to the magnetic field lines. Fig. 3 presents the $\boldsymbol{B}_{y}$ isovalues obtained by numerical simulations. Positive and negative $\boldsymbol{B}$ values reflect the direction of the vector on the $y$-axis, going up for positive values or going down when negative.

When two magnets are placed in attraction and in the absence of iron beads (Fig. 3a), the magnetic field lines go straight from one magnet towards the other, but at the edges of the magnet the magnetic field lines create a loop, ${ }^{37}$ and their density as well as $\boldsymbol{B}$ magnitude decrease. In the channel zone, $\boldsymbol{B}$ presents

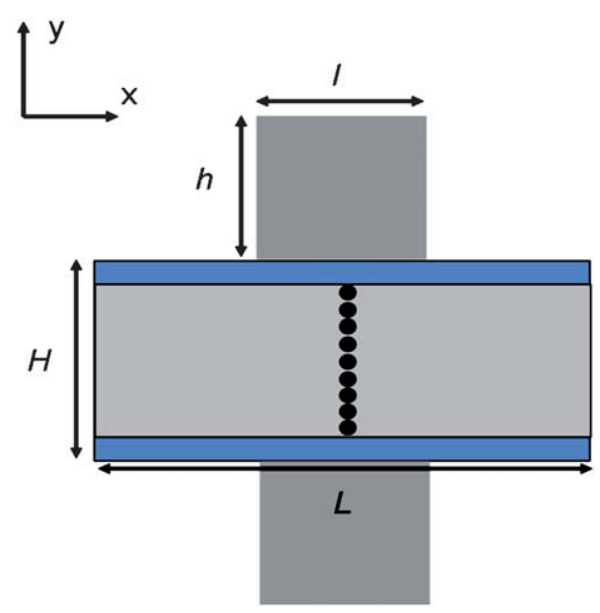

Fig. 2 Scheme of the system used for numerical simulations. The system is composed of microchannel (light grey) closed by insulating layers (blue) and surrounding by two permanent magnets (dark grey). An iron beads column (dark spot) is placed on the $y$ symmetry axis of the magnets. The whole system is placed in an air box (dark lines). $H, L, h$ and $l$ represent respectively the height of the channel, the length of the channel, the height of the magnets and their length. 


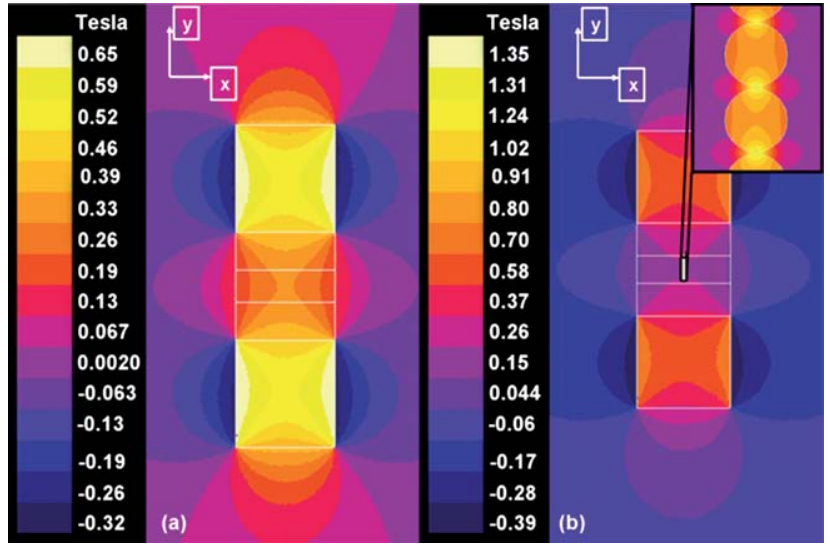

Fig. 3 Mapping of $\boldsymbol{B}$ isovalues (Tesla) $y$ component in the two considered systems: without (a) and with (b) a column of iron beads. The inset is a focus on the beads. The direction of $\boldsymbol{B}$ vectors on the $y$ axis is indicated by positive ( $\boldsymbol{B}$ vectors going up) or negative ( $\boldsymbol{B}$ vectors going down) values.

principally a $y$-component. The simulation indicates that the highest $\boldsymbol{B}$ value in the microchannel is obtained on the magnets $y$ symmetry axis and is about $0.26 \mathrm{~T}$.

In the presence of one iron bead column within the channel (Fig. 3b), the ferromagnetic beads concentrate the magnetic field lines from the external permanent magnet due to their high magnetic permeability thus amplifying the $\boldsymbol{B}$ value. Each bead behaves like a micromagnet. Indeed considering one bead, the resulting $\boldsymbol{B}$ presents a heterogeneous distribution on the $y$-axis. The highest $\boldsymbol{B}$ values are located at the bead magnetic poles and decrease until the "equator". The maximum $\boldsymbol{B}$ value was evaluated to be $1.35 \mathrm{~T}$ in the presence of iron beads, which is 6.5 times higher than the system without iron beads.

In order to evaluate where the nanoparticles are preferentially attracted, Fig. 4a presents the isovalues of the magnetic force $\boldsymbol{F}_{\boldsymbol{m a g}}$ ( $x$ component) applied to the MCSNPs in the absence of iron beads. Considering the symmetry on the $y$-axis in the centre of the channel and a dynamic case where a flow rate is continuously applied, three different situations can be determined. On

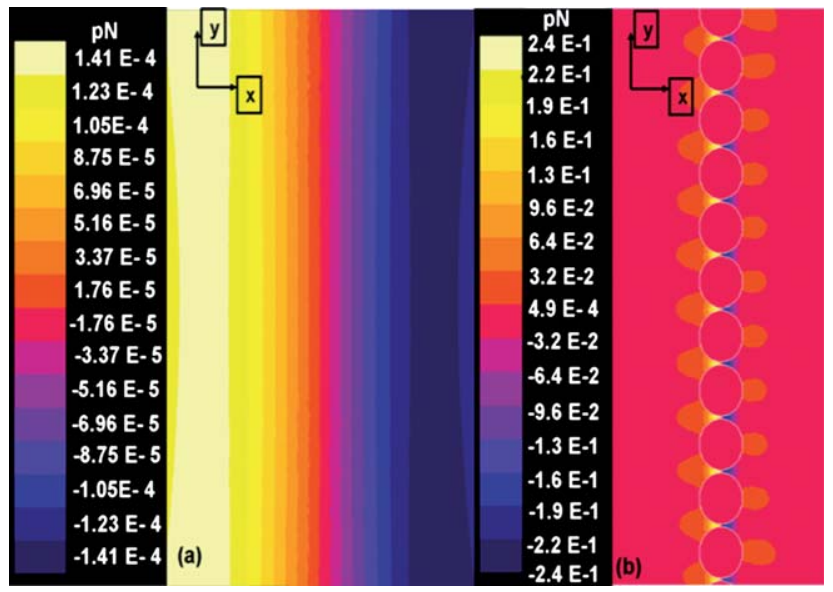

Fig. 4 Mapping of $\boldsymbol{F}_{\boldsymbol{m a g}}(\mathrm{pN})$ x component isovalues acting on a $30 \mathrm{~nm}$ MCSNPs at microchannel scale. Two situations are shown: without (a) and with (b) iron bead column. The direction of $\boldsymbol{F}_{\boldsymbol{m a g}}$ vector on $\mathrm{x}$ axis is indicated by positive (to the right) or negative (to the left) value. the left part of the $y$-symmetry axis, the MCSNPs are submitted to positive values of magnetic force, $\boldsymbol{F}_{\text {magx }}$ and $\boldsymbol{F}_{\text {drag }}$ are superimposed resulting in MCSNPs acceleration along the $x$-axis. Along the $y$-symmetry axis, $\boldsymbol{F}_{\boldsymbol{m a g x}}$ is equal to zero, the only force exerted on the $x$-axis being $\boldsymbol{F}_{\text {drag }}$, the MCSNPs slow down. On the right part $\boldsymbol{F}_{\boldsymbol{m a g} \boldsymbol{x}}$ is negative and opposite to $\boldsymbol{F}_{\text {drag. }}$. In the case where $\boldsymbol{F}_{\text {mag }}>\boldsymbol{F}_{\text {drag }}$, the MCSNPs are forced back towards the middle of the magnets. Finally, the MCSNPs are preferentially attracted and trapped in the middle of the two magnets.

In the presence of iron beads, isovalues of $\boldsymbol{F}_{\boldsymbol{m a g}}$ on the $x$-axis are distributed with a $y$ symmetry axis as well, but at a smaller scale (Fig. 4b). This axis goes through the centre of each bead. Considering one single bead, the $\boldsymbol{F}_{\text {mag }}$ distribution is heterogeneous on the $x$-axis. Positive, null and negative $\boldsymbol{F}_{\text {magx }}$ values are observed on the left, centre and right side of the $y$-symmetry axis respectively. At the plug scale, the $\boldsymbol{F}_{\text {magx }}$ maximum is located near the bead magnetic pole and is constant on the $y$-axis. The MCSNPs capture capacity should thus be maximal near the beads magnetic poles whatever their $y$ position. The magnetic force in the presence of a single column of iron beads was evaluated to be 1700 higher than without it.

These simulations results evidence the interest of integrating magnetic beads within a channel to trap magnetic nanoparticles. To further optimize the experimental device, some parameters were varied. The size of the iron beads (diameter ranging from 1 to $8 \mu \mathrm{m}$ ) has been investigated. The simulations indicate that the resulting $\boldsymbol{F}_{\boldsymbol{m a g x}}$ increases when the iron bead diameter decreases (ESI, Fig. S1 $\dagger$ ). Indeed, $\boldsymbol{F}_{\boldsymbol{m a g}}$ depends on the gradient of $\boldsymbol{B}$, and in this case, a decrease in it is observed due to the fact that the gradient is mainly expanded on the bead radius. In parallel, simulations have investigated the influence of the entire plug length on $\boldsymbol{F}_{\boldsymbol{m a g}}$. These simulations underline that with three columns of iron beads a decrease of $7 \%$ of $\boldsymbol{F}_{\text {mag }}$ is observed compared to the one achieved with one column. When increasing the plug length, the magnetic field lines will be distributed everywhere in the plug and not only concentrated through one column of beads, thus locally the magnetic field line density will decrease compared to a one-column case. These results indicate that a shorter plug should generate higher $\boldsymbol{F}_{\text {mag }}$ and thus favor nanoparticle trapping.

Finally, the nature of the beads constituting the magnetic chamber was studied. $6 \mu \mathrm{m}$ iron beads whose magnetic weight represents $99 \%$ of total weight were compared to $6 \mu \mathrm{m}$ magnetic polystyrene beads composed of magnetic iron core $(20 \%$ of total weight) encapsulated in a polystyrene shell. The resulting $\boldsymbol{F}_{\boldsymbol{m a g} \boldsymbol{x}}$ was 7 times lower using polystyrene magnetic beads highlighting the influence of the beads relative magnetic weight on $\boldsymbol{F}_{\text {mag }}$.

These numerical simulations have exemplified the ability of packed iron beads in a microchannel to increase $\boldsymbol{B}$ and $\boldsymbol{F}_{\text {mag }}$ showing that a short plug containing iron beads of small diameter may favour nanoparticles trapping.

\section{Experimental trapping and release of magnetic nanoparticles}

According to the simulations results, a short plug of micrometric magnetic beads has been formed using small diameter spherical beads $(6-8 \mu \mathrm{m})$ made of $99 \%$ iron. The choice of bead diameter was also related to the backpressure generated by the beads packed against the physical restriction. 
Fig. 5 presents the experimental results of the fluorescent MCSNPs trapping and release under a flow rate of $50 \mu \mathrm{L} \mathrm{h}^{-1}$, using permanent magnet 1 placed $5 \mathrm{~mm}$ from the channel. First, fluorescent MCSNPs were flowed through the channel in the absence of the external permanent magnet i.e. through nonmagnetized iron beads. No increase in the fluorescence signal was observed within the iron beads plug highlighting that nanoparticles were not non-specifically adsorbed on iron beads. When the external magnet was placed on the top of the microdevice (Fig. 5a), the increase in fluorescence shows that MCSNPs were progressively trapped from the entrance of the magnetic chamber. No fluorescent signal was detected after the physical restriction underlining that all the MCSNPs were trapped (0-15 s) within the iron beads plug. The magnetic chamber saturation was reached after $25 \mathrm{~s}$, the non-trapped MCSNPs flowing through the magnetic plug were detected in the channel behind the physical restriction and the fluorescent signal within the plug remains constant. The capture capacity, which is defined as the maximal amount of MCSNPs trapped using such a magnetic chamber was evaluated to $1.1 \times 10^{4} \mathrm{MCSNPs} \mu \mathrm{m}^{-3}$ and the preconcentration factor achieved was approximately 4000 .

Concerning the release step (Fig. 5 b), after the magnet's removal, only $2 \mathrm{~s}$ and a volume of $27 \mathrm{~nL}$ were necessary to elute all the nanoparticles. The absence of residual fluorescent signal in the magnetic chamber after release confirmed that the MCSNPs were not adsorbed onto iron beads even at a very high concentration and that no visible remanent magnetization persists in the iron beads magnetic plug. A good repeatability $(\mathrm{RSD}=2 \%)$ of the capture capacity was obtained for four successive fluorescent MCSNPs trapping/release sequences. These experimental results correlate with the numerical simulations. Indeed, in the absence of iron beads within the microchannel, fluorescent MCSNPs cannot be trapped with a permanent magnet even at a low flow rate, whereas the integration of packed iron beads allows for a fast and efficient trapping of $30 \mathrm{~nm}$ MCSNPs.

Similar experiments were performed using polystyrene magnetic beads instead of the iron beads and in agreement with the simulations, the capacity of capture obtained was $6.9 \times 10^{2}$ MSCNPs $\mu \mathrm{m}^{-3}$, which is 15 times lower than with the iron beads.
To further optimize this microdevice for nanoparticles trapping, the influence of the magnet size and its distance from the channel on MCSNPs trapping has been investigated.

We first evaluated the variation of $\boldsymbol{B}$, measured by a gaussmeter, for each magnet according to the distance from the magnet pole (Fig. 6a). The magnet properties are described in the method and model section. As expected, the magnet geometry has an influence on $\boldsymbol{B}$. Even if the magnetic volume is not conserved for all the magnets, $\boldsymbol{B}$ decreases with the distance from the magnet and the $\boldsymbol{B}$ value on the magnet pole surface increases with the magnet height. These results are illustrated by the highest $\boldsymbol{B}$ value obtained with magnets 1 and 3 . This can be explained by the fact that for flat magnets ( 2 and 4 ), the distance covered by the magnetic field lines outside the magnet is important compared to the one covered inside the magnet thus decreasing the magnetic field line density. Moreover, $\boldsymbol{B}$ decreases much more quickly away from magnet 3 and 4 surface compared to magnet 1 and 2 .

We have then investigated the effect of the magnet size and consequently of the applied $\boldsymbol{B}$ value on the amount of MCSNPs that could be trapped at a given distance from the magnet $(0.5 \mathrm{~cm}$ from the channel) (Fig. 6b). The real $\boldsymbol{B}$ value within the magnetic chamber has not been experimentally determined, but it was assumed that increasing $\boldsymbol{B}$ at the external magnet surface leads to an increase in $\boldsymbol{B}$ value inside the magnetic chamber until a saturation as explained later. As expected, the capture capacity varies as a function of the magnet properties. The highest nanoparticle capture capacity was achieved with magnets 1 and 2 .

Thereafter, a similar study was performed to evaluate the influence of the magnet distance from the channel on the capture capacity. The magnet producing the highest $\boldsymbol{B}$ value (magnet 1) has been used. Fig. $6 \mathrm{c}$ shows that beyond $1 \mathrm{~cm}$, increasing the distance of magnet 1 from the magnetic chamber leads to a decrease in the MCSNPs capture capacity. Simulations (data not shown) underlined that for magnet 1 placed $0.5 \mathrm{~cm}$ from magnetic chamber, the magnetic field lines were parallel in the channel zone and $\boldsymbol{B}$ was homogeneous in channel depth ( $y$-axis). One can expect that if the magnet distance increases, the magnetic field lines in the microchannel are not parallel anymore
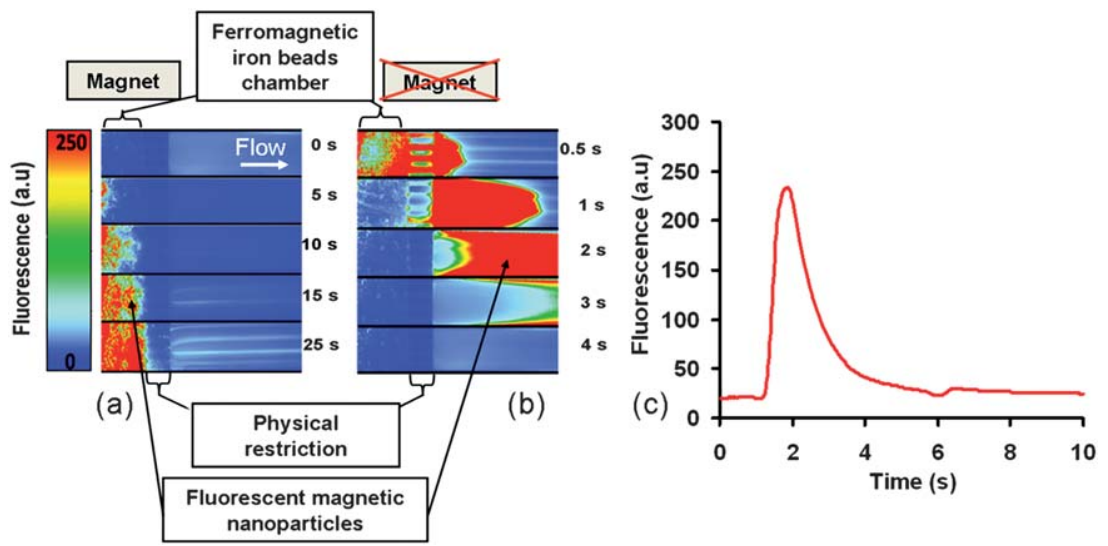

Fig. 5 Experimental trapping (a) and release (b) of fluorescent MCSNPs in a magnetic chamber of $300 \times 200 \times 30 \mu \mathrm{m}^{3}$ using inverted microscope imaging (top view). The flow rate was fixed at $50 \mu \mathrm{L} \mathrm{h}^{-1}$ and the initial concentration of MCSNPs was put at $6.2 \times 10^{13} \mathrm{MCSNPs} / \mathrm{mL}$. (c) Fluorescent MCSNPs peak profile obtained after magnet removal, the detection window $100 \mu \mathrm{m}$ length and $200 \mu \mathrm{m}$ width was arbitrary placed with IRIS software at $100 \mu \mathrm{m}$ from physical restriction ending. 

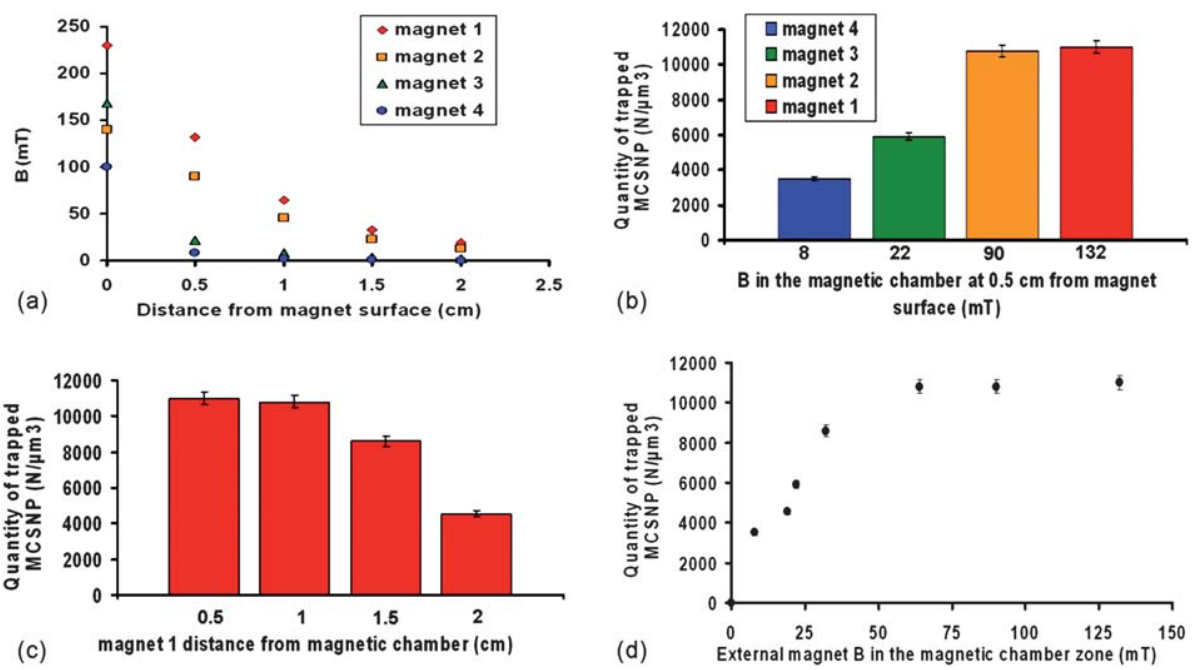

Fig. 6 (a) Evolution of $\boldsymbol{B}$ according to the distance from magnet surface using a gaussmeter. (b) Magnet influence on MCSNPs trapping efficiency at 0.5 $\mathrm{cm}$ from magnetic chamber. (c) Influence of magnet 1 distance from magnetic chamber on MCSNPs trapping efficiency. (d) Summary of trapped MCSNPs quantity variation as a function of external permanent magnet $\boldsymbol{B}$ in the magnetic chamber area.

and thus $\boldsymbol{B}$ becomes heterogeneous in the channel zone on the $y$-axis leading to a decrease of bead magnetization with the channel depth. Finally, both magnet shape and distance were varied in order to study directly the influence of $\boldsymbol{B}$ on the MCSNPs trapping. Fig. 6d presents the MCSNPs capture capacity according to the external magnet $\boldsymbol{B}$ value in the channel zone. The quantity of trapped MCSNPs increases with $\boldsymbol{B}$ until reaching a plateau. We identify that, in the present conditions, maximal MCSNPs trapping capacity will be obtained by applying an external $\boldsymbol{B}$ value of about $50 \mathrm{mT}$ in the magnetic chamber. Maximal MCSNPs trapping capacity might be due to iron beads saturation magnetization which is the state reached when the increasing of external $\boldsymbol{B}$ has no more effect on material magnetization; all the spins of iron bead magnetic domains are aligned. Maximal trapping capacity could also be attributed to the saturation of MCSNP volume occupancy where $\boldsymbol{F}_{\text {mag }}>\boldsymbol{F}_{\text {drag }}$.

Finally, in order to perform high throughput analysis, the influence of flow rates on MCSNPs trapping kinetics has been studied using magnet 1 at $0.5 \mathrm{~cm}$ from the magnetic chamber

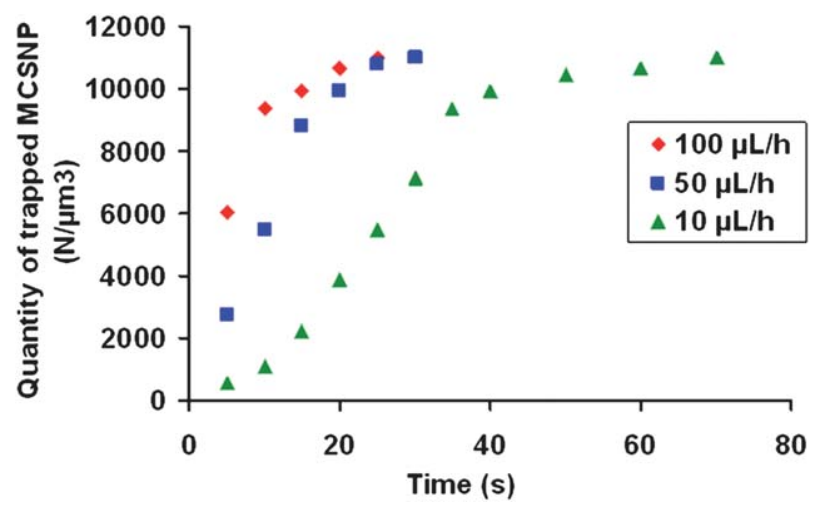

Fig. 7 Influence of flow rates on trapped MCSNPs occupancy in the magnetic chamber. The quantity of MCSNPs was evaluated by measuring the surface of the fluorescent signal into the magnetic chamber using microscope imaging.
(Fig. 7). Three different flow rates have been investigated: 10, 50 and $100 \mu \mathrm{L} \mathrm{h}^{-1}$. The capacity of capture is not affected by the flow rate (data not shown) illustrating that the magnetic force generated is still higher than the drag force. As expected the higher the flow rate, the faster the capture capacity saturation is reached. Saturation of the magnetic plug was obtained after 15, 25 and $50 \mathrm{~s}$ using 100,50 and $10 \mu \mathrm{L} \mathrm{h}^{-1}$ flow rate respectively. No higher flow rate has been used to avoid clogging of the physical restriction.

\section{Conclusion}

We have successfully developed a microdevice integrating a functional magnetic chamber consisting of packed micrometric iron beads dedicated to $30 \mathrm{~nm}$ magnetic nanoparticles trapping.

Due to the small size of the nanoparticles and their low content of magnetic material, high magnetic flux gradient is required to magnetically trap these nanometric objects. In this way, ferromagnetic micrometric beads were used to concentrate magnetic field lines produced by an external magnet and to locally increase $\boldsymbol{B}$ and therefore $\boldsymbol{F}_{\text {mag }}$. Finite element numerical simulations have been carried out to help develop the device. They have shown the local increase in $\boldsymbol{B}$ and $\boldsymbol{F}_{\boldsymbol{m a g}}$ in the presence of iron beads as well as their distribution. The proof of concept of this microdevice to easily and efficiently trap and release fluorescent $30 \mathrm{~nm}$ colloidal MCSNPs was demonstrated. Furthermore, the influence of ferromagnetic bead nature and magnet on $\boldsymbol{F}_{\text {mag }}$ gain respectively as well as the flow rate on MCSNPs trapping kinetics was investigated. Due to their colloidal behaviour and their tuneable surface chemistry, future developments are in progress to combine nanoparticle advantages and their efficient trapping in this microdevice.

\section{Acknowledgements}

This work was supported by the French research agency ANR SOLUDIAG ANR-07-BLAN-02660 and by a Swiss National 
Science Foundation grant entitled "Supramolecular phases for protein adsorption" (Grant No 404740-117321). Thanks go to Dr Jacques Josserand for his assistance in the numerical simulations.

\section{References}

1 K. S. Kim and J. K. Park, Lab Chip, 2005, 5, 657-664.

2 J. W. Choi, K. W. Oh, J. H. Thomas, W. R. Heineman, H. B. Halsall, J. H. Nevin, A. J. Helmicki, H. T. Henderson and C. H. Ahn, Lab Chip, 2002, 2, 27-30.

3 S. Y. Yang, K. Y. Lien, K. J. Huang, H. Y. Lei and G. B. Lee, Biosens. Bioelectron., 2008, 24, 855-862.

4 J. Do and C. H. Ahn, Lab Chip, 2008, 8, 542-549.

5 M. A. M. Gijs, F. Lacharme and U. Lehmann, Chem. Rev., 2010, 110, $1518-1563$.

6 N. Pamme, Lab Chip, 2006, 6, 24-38.

7 M. Slovakova, N. Minc, Z. Bilkova, C. Smadja, W. Faigle, C. Futterer, M. Taverna and J. L. Viovy, Lab Chip, 2005, 5, 935-942.

8 F. Lacharme, C. Vandevyver and M. A. M. Gijs, Anal. Chem., 2008, 80, 2905-2910.

9 S. Bronzeau and N. Pamme, Anal. Chim. Acta, 2008, 609, 105-112.

10 A. Sinha, R. Ganguly and K. I. Puri, J. Magn. Magn. Mater., 2009, 321, 2251-2256.

11 M. A. Hayes, N. A. Polson, A. N. Phayre and A. A. Garcia, Anal. Chem., 2001, 73, 5896-5902.

12 Z. H. Fan, S. Mangru, R. Granzow, P. Heaney, W. Ho, Q. Dong and P. R. Kumar, Anal. Chem., 1999, 71, 4851-4859.

13 L. Korecka, Z. Bilkova, M. Holeapek, J. Kralovsky, M. Benes, J. Lenfeld, N. Minc, R. Cecal, J. L. Viovy and M. Przybylski, J. Chromatogr. B, 2004, 808, 15-24.

14 M. Abonnenc, A.-L. Gassner, J. Morandini, J. Josserand and H. H. Girault, Anal. Bioanal. Chem., 2009, 395, 747-757.

15 T. Deng, M. Prentiss and G. M. Whitesides, Appl. Phys. Lett., 2002, 80, 461-463.

$16 \mathrm{M} . \mathrm{Bu}, \mathrm{T}$. B. Christensen, K. Smistrup, A. Wolff and M. F. Hansen, Sens. Actuators A, 2008, 145, 430-436.

17 K. Smistrup, O. Hansen, H. Bruus and M. F. Hansen, J. Magn. Magn. Mater., 2005, 293, 597-604.
18 E. Verpoorte, Lab Chip, 2003, 3, 60-68.

19 J. Won, M. Kim, Y. W. Yi, Y. H. Kim, N. Jung and T. K. Kim, Science, 2005, 309, 121-125.

20 B. H. Hui, Z. J. Tsao, J. J. Wang and F. Y. Yu, Anal. Chem., 2008, 80, 7029-7035.

21 C. H. Chiou, Y. Y. Huang, M. H. Chiang, H. H. Lee and G. B. Lee, Nanotechnology, 2006, 17, 1217-1224.

22 J. Dobson, Nat. Nanotechnol., 2008, 3, 139-143.

23 T. Rheinländer, R. Kötitz, W. Weitschies and W. Semmler, J. Magn. Magn. Mater., 2000, 219, 219-228.

24 M. Donolato, M. Gobbi, P. Vavassori, M. Leone, M. Cantoni, V. Metlushko, B. Ilic, M. Zhang, S. X. Wang and R. Bertacco, Nanotechnology, 2009, 20, 385501-385507.

25 T. B. Jones, Electromechanics of particles, 1995, Cambridge University Press.

26 J. C. McDonald and G. M. Whitesides, Acc. Chem. Res., 2002, 35, 491-499.

27 J. C. McDonald, D. C. Duffy, J. R. Anderson, D. T. Chiu, H. K. Wu, O. J. A. Schueller and G. M. Whitesides, Electrophoresis, 2000, 21, 27-40.

28 R. Massart, J. C. Bacri, R. Perzynski and D. Salin, J. Magn. Magn. Mater., 1986, 62, 36-46.

29 N. Fauconnier, A. Bee, J. Roger and J. N. Pons, Prog. Colloid. Polym. Sci., 1996, 100, 212-216.

30 Y. Lu, Y. Yin, B. T. Mayers and Y. Xia, Nano Lett., 2002, 2, 183-186.

31 V. Maurice, T. Georgelin, M. J-Siaugue and V. Cabuil, J. Magn. Magn. Mater., 2009, 321, 1408-1413.

32 T. Georgelin, V. Maurice, B. Malezieux, J.-M. Siaugue and V. Cabuil, J. Nanopart. Res., 2010, 12, 675-680.

33 P. Campbell, Permanent Magnet Materials and their Application, ed. Cambridge University Press, 1996, pp 7-8.

34 G. P. Hatch and R. E. Stelter, J. Magn. Magn. Mater., 2001, 225, $262-$ 276.

35 J. Liu, E. M. Lawrence, A. Wu, M. L. Ivey, G. A. Flores, K. Javier, J. Bibette and J. Richard, Phys. Rev. Lett., 1995, 74, 2828-2831.

36 P. S. Doyle, J. Bibette, A. Bancaud and J.-L. Viovy, Science, 2002, 295, 2237.

37 A.-L. Gassner, M. Abonnenc, H. X. Chen, J. Morandini, J. Josserand, J. S. Rossier, J.-M. Busnel and H. H. Girault, Lab Chip, 2009, 9, 2356-2363. 